\title{
10
}

\section{A Proposal for Composing Software Design Process}

\author{
Sumio Mochizuki" and Takuya Katayama ${ }^{b}$ \\ ${ }^{a}$ Shukutoku Junior College, 1150-1, Fujikubo, Miyoshi-cho, Iruma-gun, Saitama-ken, 354, \\ Japan (Previous affiliation: Mitsubishi Space Software Co.) \\ ${ }^{b}$ Department of Computer Science, Faculty of Engineering, Tokyo Institute of Technology \\ 2-12-1, Ookayama, Meguro-ku, Tokyo, 152, Japan
}

\begin{abstract}
We described a real industrial process in terms of HFSP(Hierarchical and Functional Software Process).[1] After analyzing design process of our experienced engineers, we discovered two describing methods, that is, object-centered process and phase-centered process which can respectively show the design process from their different points of view. It can be considered that they extracted generic object-centered process from their rich design experiences, and applied it to real design works, converting it into the phase-centered process suitable to design the objective system. In this paper we described the two process description methods and the way how to convert object-centered process into phase-centered process on the basis of object transition. We clarified the method how to compose the phases for a concrete system design and how to execute object-centered process in a phase.
\end{abstract}

Keyword Codes : D. $2.10 ;$ K. $3.2 ;$ H. 0

Keywords : Software Engineering, Design; Computer and Information Science Education ; Information Systems, General

\section{INTRODUCTION}

We wanted to describe formally our real design process. In the research, after analyzing design process of our experienced engineers, we discovered two describing methods: Objectcentered process and phase-centered process. [2] [3] Comparing them, we discovered that transition of objects is the important key. We clarified the method how an experienced engineer design a concrete system, applying these models.

\section{PRODUCTION AND TRANSITION OF OBJECTS}

Various objects are produced in the middle of the design works. Some objects produced are modified by the next process. These are the temporary products, which we call intermediate objects. The final results, which we call final objects, are retained until the design works are finished, and they are included as the parts of the fundamental design document. After observing real design processes, we discovered there are the following two types of transition.

a. Type I transition. - Input objects are converted to the output objects of different kind.

b. Type II transition. - An input object and its output object are of the same kind.

In designing a large-scaled system, some of the objects are produced repeatedly. Converting their rough contents into detailed contents, the designer can get consistent final objects. Fig. 1 shows the transition of object groups in accordance with execution of the first level process for designing the Data Acquisition System for checkout of artificial spacecraft (DAS shortly), as a real example of Type II transition. In this figure the process is executed from left side to right side, and the transition of the object group is shown by three rough state. Arrow shows Type II transition of each object between the processes.

\section{TWO MODELS FOR DESCRIBING DESIGN PROCESS}

In the past research, we analyzed, described and evaluated a real fundamental design process for a practical system (DAS), using HFSP. [2] [3] We discovered the following two models. 


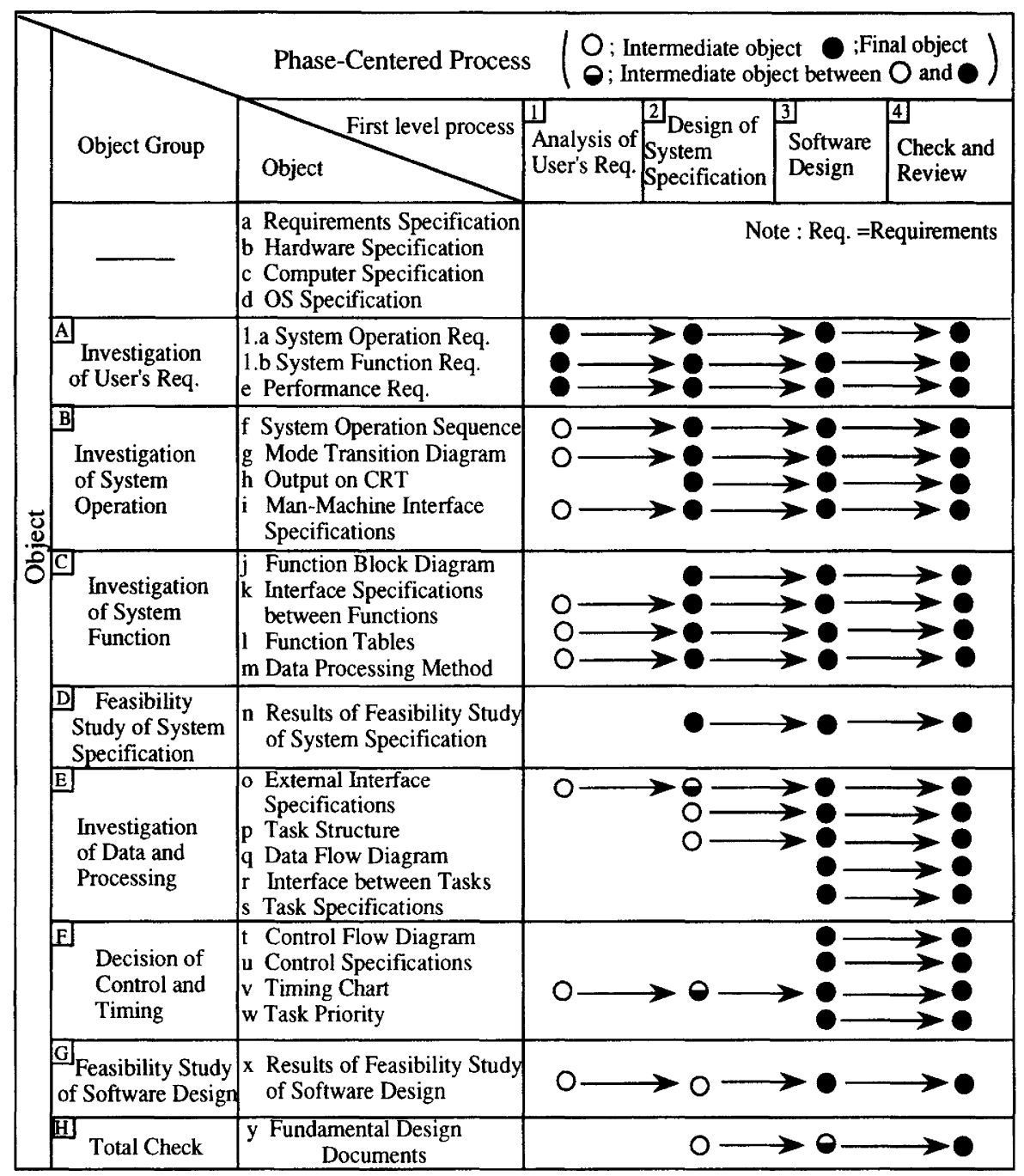

Figure 1 Transition diagram of the objects by executing first level phase-centered process for designing the practical system DAS. 


\subsection{Object-centered process}

In the fundamental design of a practical system, many objects of the same kind which are produced through type II transition. Object-centered process renames and treats objects of the same kind as a single object. After this renaming, the remaining objects are of the different kind, and these objects could be processed through type I transition. For the DAS process, collecting and classifying these objects into the groups in accordance with their contents, we get the eight object groups in Fig.1. The first level object-centered process, which produces these object groups, are shown in Fig.2. These object-centered processes are executed from upper to lower in accordance to the relations between objects. There are only type I transitions in this objectcentered process. This object-centered process can express concisely and comprehensibly static relations between the objects and can describe various design procedures of the similar systems in an application area as a common process, therefore this might be called a generic model.

\section{2 Phase-centered process}

The object-centered process places importance on generality and static relations between objects. On the other hand the phase-centered process intends to describe the real design processes which could be enacted. In designing a very simple system, the contents of the objects are also simple, and we can enact its object-centered process as it is. How ever, in the case of designing a large-scaled system, we have to produce many objects having deep and complicated logical structure. As the objects in object-centered process are generated through object refinement repeating process, the object-centered process cannot be enacted directly unless it is unfolded into an executable process as shown in Fig.2, where such refinement is explicitly described. From the standpoint of project management, design works should necessarily be divided into phases which are the first level phase-centered processes. Here we assume the following characteristics for the phases. (1) Objects which have close relation with each other are defined in one phase. (2) The purpose of design works in each phase is to define the final objects which are allotted to the phase in the project plan. (3) Repeating and preliminary execution of processes are necessary in a phase for designing a large-scaled system. (4) Final objects are defined sequentially in accordance with the execution of phases. There is no redoing of phases in principle. (5) Phase has an interface with project management, including review of schedule, cost, and quality.

\section{DETERMINING PHASES}

In this and next sections we propose a method of composing enactable phase-centered process from an object-centered process.

\subsection{Determining fundamental phases}

Observing the object groups, we can find that there are two types of object groups. One consists of the object groups which have close relations between them and should be defined in one phase to avoid redoing in design w orks. The other consists of the object groups which can be defined in each different phase. As the results, we get the phases which define object gorups mentioned before. We call them fundamental phases. Fig. 1 shows an example in which some object groups are defined in one phase. In the figure the final objects that are included in Investigation of System Operation group, Investigation of System Function group and Feasibility Study of System Specitication group are defined in Design of System Specification process. These objects in Investigation of System Operation group and Investigation of System Function group have close relations each other, as parts of the system specifications. Changes of objects in one object group may affect the objects in the other object group, and large redoing may be needed. Therefore the final objects in those two object groups are needed to be defined in one phase. Similarly the objects in Investigation of Data and Processing group and Decision of Control and Timing group define software design totally, and have close relations each other. Therefore their final objects are to be defined in one phase. On the other hand the other object groups such as Investigation of U'ser's Requirements and Total Check are defined in each different phase. After the investigation we get phases and call those phases fundamental phases, which are shown in Table 1.

\subsection{Defining phases}

When a designer designs a concrete system, he composes appropriate phases on the basis of fundamental phases. At first he describes object-centered process correspondent to each funda- 


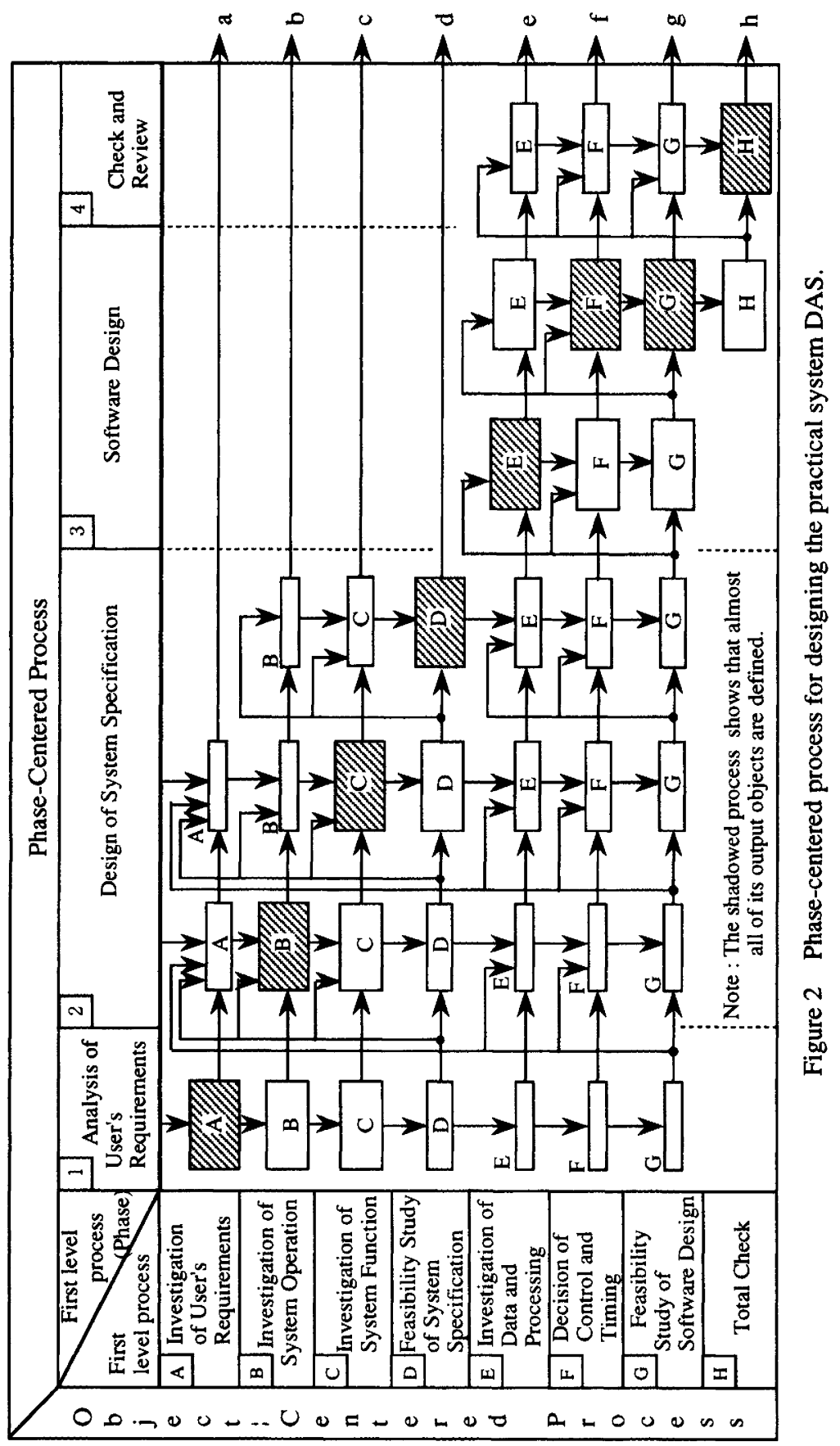


mental phase as shown in Fig. 3. That is, each fundamental phase is given objectcentered process which produces the object groups allotted to the fundamental phase in Table 1.

Furthermore, the fundamental phases are evaluated by their degree of difficulty in applying them to the objective system. Though it might be hard to measure quantitatively the degree of difficulty, their appropriateness could be evaluated, as follows.

(1) Weight of fundamental phases

We denote the weight $w(a)$ which expresses degree of difficulty of process " $a$ " in objectcentered process.

This w(a) cannot be defined precisely, but the experienced designer can define it experientially from scale and complexity of the system. A fundamental phase includes processes, and its weight is given by summing up the weights of the included processes.

(2) Composition of phases

There are upper limit value Wmax and lower limit value Wmin for the weight of an executable phase. When the weight of the fundamental phase $W(q)$ exceeds those values, the fundamental phase is regarded to be inappropriate for real design works. The appropriate phase for real design works are composed on the basis of fundamental phase as follows;

a. $W \min <W(q)<W \max$. . The design works included in the fundamental phase $q$ are appropriate, and it can be used as the phase for real design.

b. Wmax $<W(q)$. ' Weight of the fundamental phase $q$ is too difficult. However, as fundamental phases cannot be divided to smaller ones, the fundamental phase $q$ is repeated, that is, phase $q(1)$, phase $q(2)$, etc. and intermediate objects are obtained gradually.

c. $W(q)<W m i n$. Phase is the objective of project management. The design works included in the fundamental phase $q$ are so small that project management for it is redundant. Therefore some neighboring phases should be combined into one phase which is shown in Table 1 so that its size is appropriate to management works.

\section{COMPOSING PHASE-CENTERED PROCESS}

In the previous sections, we could decide the phases, the objects defined in each phase, and the processes which define the final objects in the phases. The processes were executed repeatedly, and executed preliminarily in a phase as follows. 
5.1 Repetition of processes in the phase

In case of designing a simple system, the objects allotted to each phase can be defined only by executing the processes included in each phase without repetition. In case of designing a practical system, the objects, which are to be defined in a phase, cannot be defined completely by executing only once the processes in the phase. In this case the processes are repeated and executed successively in the phase, until output objects of the phase are defined finally.

\subsection{Preliminary execu-} tion of the processes in a phase

In order to define the final objects in a phase, some processes in the later phase are preliminarily executed for checking and reviewing if the obtained objects are reasonable. We call this checking processes "Preliminary execution of the processes".

Fig. 2 shows the real design process of practical system DAS as an example of the phasecentered process. The phases are shown in the upper column, which are executed from left to right. The processes in a phase are executed from top to bottom in a column, and the next right column are executed. In this phase-centered process, there are repeats of three times in Design of System Specification phase, and repeats of two times in Sottware Design.

\section{CONCLUSION}

In this paper we described two process description methods: object-centered process and phase-centered process. Furthermore, we described the method how to convert object-centered process into phase-centered process on the basis of object transition. After analyzing the objects produced in the DAS design, we clarified the method how to compose the phases for a concrete system design and how to execute object-centered process in the phases.

\section{REFERENCES}

1. Katayama T., "A Hierarchical and Functional Software Process Description and its Enaction", Proceeding of the 11th International Conference on SoftwareEngineering, 1989, pp. 343-352.

2. Mochizuki S., Yamauchi A., Katayama T., "Describing and Evaluating Fundamental Design Process of Checkout System for Artificial Spacecraft by Process Model HFSP", Transactions of Information Processing Society of Japan, Vol. 33, No. 5, May, 1992, pp. 691-706.

3. Mochizuki S., Yamauchi A., Katayama T., "Two Models for Describing Software Design Process: Object-Centered Model and Phase-Centered Model", Proceedings, the 5th International Conference on software Engineering and Knowledge Engineering, June, 1993, pp. 291-295. 